\title{
Experimentelle Hämatologie und klinische Immunologie
}

\section{Experimental Hematology and Clinical Immunology}

\author{
U. Göbel ${ }^{1}$ \\ T. Lehrnbecher ${ }^{1}$ \\ T. Niehues ${ }^{1}$ \\ T. Klingebiel ${ }^{2}$ \\ N. Wagner ${ }^{3}$ \\ G. Ganser ${ }^{4}$
}

Die hämatopoetische Funktion des Knochenmarks wurde bereits im Jahr 1868 von dem Königsberger Pathologen Ernst Neumann in einer Kurzmitteilung „Ueber die Bedeutung des Knochenmarkes für die Blutbildung“ [19] beschrieben. Die nachfolgenden Forschungen vieler $\mathrm{Ar}$ beitsgruppen mündeten letztendlich in die Stammzelltheorie von Metcalf ein, der die wesentlichen Erkenntnisse zur Hämatopoese und deren Steuerung durch Zytokine mittels semisolider Kulturen generierte [18].

Stand bei Neumann und seinen Zeitgenossen die Bildung der roten Blutkörperchen im Vordergrund ihrer Forschungen, so sind danach vorrangig die Leukozyten ins Zentrum der experimentellen Hämatologie als auch anderer Fachrichtungen gerückt [20]. Obwohl die Leukozyten zahlenmäßig nur einen geringen Teil der in den Gefäßen zirkulierenden zellulären Blutbestandteile ausmachen, sind ihre vielfältigen physiologischen Funktionen hierfür verantwortlich, die in der Erkennung von „Selbst“ und „Fremd“ zusammengefasst werden können.

Der Erhalt der körpereigenen Integrität gegenüber eindringenden Mikroben ist dabei nur eine, aber sehr wichtige Teilfunktion, der sich die Immunitätsforschung und die Infektiologie in komplementärer Weise widmen. Während die Erforschung von angeborenen oder erworbenen Defekten der Leukozyten am Anfang der pädiatrischen Immunologie stand, wurden zwischenzeitlich auch die Immunregulationsstörungen als wichtiges Forschungsgebiet erkannt und damit eine Brücke zur pädiatrischen Rheumatologie geschlagen. Umgekehrt kann eine maligne Entartung hämatologischer Zellen auf jeder Reifungsstufe der Hämatopoese stattfinden und stellt unter dem umfassenden Oberbegriff der Leukämie die häufigste onkologische Erkrankungsgruppe im Kindesalter dar.

Die genannten Forschungsrichtungen haben zu eigenständigen Fachgesellschaften geführt, deren Mitglieder sich regelmäßig zu Fachtagungen treffen und die in spezialisierten Fachzeitschriften veröffentlichen. In den zurückliegenden Jahrzehnten haben sich nun zunehmend Überschneidungen herausgestellt, die ihre Begründung in den Erkenntnissen der experimentellen Hämatologie haben. Die Verfeinerung der diagnostischen Verfahren hätte ohne die Generierung monoklonaler Antikörper durch den Immunologen und Nobelpreisträger Georges Köhler [16] nicht den heutigen Stand erreicht. Und ohne die Entdeckung des HLA-Systems durch den ebenfalls mit einem Nobelpreis ausgezeichneten Jean Dausset $[3,4]$ wären keine erfolgreichen Transplantationen von Knochenmark oder Blutstammzellen zur lebensrettenden Therapie einer schweren aplastischen Anämie, von schweren kombinierten Immundefekten und von rezidivierten Leukämien möglich geworden.

War früher in der konditionierenden Vorbehandlung der Wirkeffekt bei der Behandlung von Leukämien durch eine Knochenmarktransplantation gesehen worden, haben spätere Untersuchungen gezeigt, dass das mit den transplantierten Stammzellen übertragene Immunsystem des Spenders entscheidend zur Kontrolle der residualen Leukämiezellen beiträgt. Somit handelt es sich bei der onkologisch indizierten hämatopoetischen Blutstammzelltransplantation auch um eine immunologische Therapie. Umgekehrt gibt es Ansätze, immunologische Erkrankungen mit Immundysregulation durch autologe Stammzelltransplantationen zu behandeln [21].

Für die Erforschung der experimentellen Grundlagen der Blutstammzelltranplantation und die systematische klinische Umsetzung der gewonnenen Erkenntnisse hat Donnall Thomas [22] den Nobelpreis für Medizin erhalten. Hierdurch ist erstmals ein neuartiges Therapiekonzept in dieser hervorragenden Weise ausgezeichnet worden, das nur durch die Vernetzung von unterschiedlichen Forschungsrichtungen möglich wurde.

Diese Aneinanderreihung von wichtigen Meilensteinen der experimentellen Hämatologie und klinischen Immunologie lässt nicht erkennen, wie viele kleine Einzelschritte zwischen jedem Meilenstein zu bewältigen waren. Noch weniger wird erkennbar, wie viele Patienten ohne den ersehnten Erfolg behandelt wurden oder trotz aller Fortschritte die Nichterfüllung ihrer Hoffnungen noch immer erleben müssen.

Die alltägliche Konfrontation mit dieser Realität hat an vielen Behandlungseinrichtungen für krebskranke Kinder Forschungslabore mit unterschiedlichen Zielrichtungen entstehen lassen, was meist nur durch die Zuweisung erheblicher Spendenmittel möglich war [10]. Die interdisziplinäre Nutzung der zum Teil sehr aufwendigen apparativen und personellen Ausstattungen führt zu neuen Kooperationen, die auch anderen Patientengruppen zugute kommen. Insofern ist $\mathrm{zu}$ 
hoffen, dass auf dem langen und mühsamen Weg der vielen kleinen Einzelschritte neue Erkenntnisse in der Diagnostik und Therapie für möglichst viele Patienten generiert werden können. Beispiele dieser interdisziplinären Kooperation sind die Jahrestagungen der Kind-Philipp-Stiftung für Leukämieforschung, die seit 21 Jahren junge Forscher unterschiedlicher Fachrichtungen in Wilsede/Lüneburger Heide zusammenführen und deren Beiträge als Abstracts in dieser Zeitschrift regelmäßig publiziert werden [11-13]. Die gleichfalls regelmäßig in zeitlich größeren Abständen stattfindenden Reisensburg Symposien dienen dahingegen dem Gedankenaustausch international führender Forscher mit deutschen pädiatrischen Hämatologen und Onkologen, um neue Entwicklungen zu diskutieren und zu fördern. So hatte die letzte Tagung die Immuntherapie bei Kindern zum Inhalt, die aus verschiedenen Forschungsrichtungen diskutiert wurde; beispielsweise waren die natürlichen Killer-Zellen mit ihrer Alloreaktivität nach allogener Blutstammzelltransplantation sowie als zelluläre Therapeutika, monoklonale Antikörper zur Tumortherapie oder die Tumorvakzination vier der acht Themenschwerpunkte [14].

Einen direkten Praxisbezug haben die Supplemente zur antiinfektiösen Prophylaxe und Therapie bei Kindern und Jugendlichen mit zytostatischer Therapie $[2,17]$. Hier wurde erstmals versucht, Standards für die tägliche Arbeit am Krankenbett für den deutschsprachigen Raum bereitzustellen, die einer ungeprüften Polypragmasie vor allem in lebenskritischen Situationen entgegenwirken und durch evidenzbasierte Maßnahmen das Eintreten von Komplikationen verhindern bzw. deren Behandlung verbessern sollen.

Mit Band 36 der Forschungsergebnisse der pädiatrischen Onkologie, Hämatologie und Immunologie ist dieser Entwicklung seitens der Zeitschrift Klinische Pädiatrie Rechnung getragen worden [7]; die Früherkennung schwerer kombinierter Immundefekte [9], die Wegener-Granulomatose [6] und die immunologische Immunrekonstitution nach Ende der ALL-Therapie [1] waren die Themen der immunologischen Arbeiten. Von sehr viel weiter reichender Bedeutung ist jetzt die Veröffentlichung der interdisziplinären S2-Therapieleitlinie der Juvenilen Idiopathischen Arthritis durch die Gesellschaft für Kinder- und Jugendrheumatologie, an der acht weitere wissenschaftliche Fachgesellschaften, Verbände und Vereinigungen mitgewirkt haben [8]. Auf diese Weise konnten auch die Konzepte der Physiotherapeuten, der Psychologen und Sozialpädagogen sowie der Selbsthilfeorganisationen und Eltern mit eingebracht werden. Besser kann der Wille zur Kooperation und Vernetzung bei der Behandlung einer wichtigen Patientengruppe nicht dargestellt werden. Synergien werden an Einzelprojekten wie der Apoptose aus experimenteller Sicht bei Kindern mit akuter lymphatischer Leukämie [23] und juveniler idiopathischer Arthritis [5] erkennbar, die über die gemeinsame Nutzung von verfügbaren Ressourcen hinausgehen. Die Pilotstudie zur Rekonstitution Zytomegaliespezifischer T-Zellen nach allogener Blutstammzelltransplantation beinhaltet eine immunologische Fragestellung bei einem onkologischen Patientengut und beschreibt das proof of principle vor Aktivierung einer prospektiven multizentrischen Studie [15]. Die Entscheidung zur Therapie bei Uveitis mit konventioneller Immunsuppression oder TNF $\alpha$-blockierenden Agenzien trifft der Augenarzt, während der pädiatrische Rheumatologe/ Immunologe/Hämatologe/Allgemeinpädiater die therapiebegleitende allgemeine Diagnostik durchführt; insofern ist eine interdisziplinär erstellte Übersicht zum aktuellen Wissensstand für diese klinische Situation besonders hilfreich für ein auf Evidenz basiertes Vorgehen [24].

Es ist zu hoffen, dass möglichst viel dieser mühsam erarbeiteten Einzelaspekte sich in Zukunft wieder so zusammenfügen, dass ein weiterer Meilenstein in der Geschichte der experimentellen Hämatologie erreicht werden kann, der letztendlich unseren Patienten zugute kommt.

\section{Literatur}

1 Calaminus G, Hense B, Laws HJ et al. Diphtherie- und Tetanusantikörper bei Kindern mit akuter lymphatischer Leukämie nach Beendigung der Therapie mit dem Co-ALL-05/92-Protokoll. Klin Pädiatr 2007; 219: 355-360

2 Creutzig $U$, Belohradsky BH Hrsg. Infektionsprophylaxe bei hämatologisch-onkologischen Patienten in der Pädiatrie. Klin Pädiatr 2001; 213: A1-A144

3 Dausset J, Brecy H. Identical nature of the leucocyte antigens detectable in monozygotic twins by means of immune iso-leuco-agglutinins. Nature 1957; 180 (4599): 1430

4 Dausset J, Rapaport FT. Role of ABO erythrocyte groups in human histocompatibility reactions. Nature 1966; 209 (5019): 209-211

5 Feyen 0 , Telieps T, Schmitz I et al. CD95 mediated apoptosis in synovial fluid and peripheral blood MNCs of JIA patients: Disease specific caspase-8 cleavage and FlipShort overexpression. Klin Pädiatr 2008; 220: $358-364$

6 Fiebig B, Hedrich CM, Heubner G et al. Wegener-Granulomatose bei pädiatrischen Patienten. Klin Pädiatr 2007; 219: 348-354

7 Göbel U, Jürgens H Hrsg. Forschungsergebnisse der pädiatrischen Onkologie, Hämatologie und Immunologie 36. Klin Pädiatr 2007; 219: 303-305

8 Guellac N, Niehues T. Interdisziplinäre S2-Therapieleitlinie der Juvenilen Idiopathischen Arthritis. Klin Pädiatr 2008; 220: 392-402

9 Hennewig $U$, Schulz A, Adams $O$ et al. Hinweis auf schweren kombinierten Immundefekt durch Eosinophilie und Lymphopenie bei Säuglingen mit Rotavirusinfektion. Klin Pädiatr 2007; 219: 343-347

10 Herold $R$, Reiche $R$, Creutzig $U$ et al. Stand und Bewertung der Strukturen und Ausstattung pädiatrisch-onkologisch-hämatologischer Kliniken. Klin Pädiatr 2007; 219: 380-390

11 Kabisch $H$ Hrsg. XIX. Jahrestagung der Kind-Philipp-Stiftung für Leukämieforschung, 31. März-2. April 2006. Klin Pädiatr 2006; 218: 185-200

12 Kabisch $H$ Hrsg. XX. Jahrestagung der Kind-Philipp-Stiftung für Leukämieforschung, 6.-9. Juni 2007. Klin Pädiatr 2007; 219: 181-198

13 Kabisch H Hrsg. XXI. Jahrestagung der Kind-Philipp-Stiftung für Leukämieforschung, 11.-14. Juni 2008. Klin Pädiatr 2008; 220: 198-214

14 Klingebiel T, Bader P, Hollatz G et al. Immunotherapy in children: Report from the Reisensburg-Symposium October 20-22, 2004 and recent advances. Klin Pädiatr 2006; 218: 355-365

15 Koehl U, Dirkwinkel E, Koenig $M$ et al. Reconstitution of cytomegalovirus specific $\mathrm{T}$ cells after pediatric allogeneic stem cell transplantation: Results from a pilot study using a multi-allele CMV tetramer group. Klin Pädiatr 2008; 220: 348-352

16 Köhler G. Derivation and diversification of monoclonal antibodies. Science 1986; 233: 1281-1286

17 Laws HJ, Lehrnbecher T Hrsg. Therapie von Infektionen in der Kinderonkologie. Klin Pädiatr 2005; 217: S1-S174

18 Metcalf $D$. Hemapoietic colonies-in vitro cloning of normal and leukemic cells. Springer, Berlin, Heidelberg, New York; 1977

19 Neumann E. Ueber die Bedeutung des Knochenmarkes für die Blutbildung. Centralbl Med Wiss 1868; 6 (44): 689

20 Neumann HA, Klinger $Y$. Knochenmark und Stammzelle - Der Kampf um die Grundlagen der Hämatologie. Blackwell Wissenschafts-Verlag, Berlin; 1994, ISBN 3-89412-192-0

21 Passweg J, Tyndall A. Autologous stem cell transplantation in autoimmune diseases. Semin Hematol 2007; 44: 278-285

22 Thomas ED, Ferrebee JW. Irradiation and marrow transplantation: studies in Cooperstown. Lancet 1960; 1 (7137): 1289-1290

23 Troeger A, Glouchkova L, Escherich $G$ et al. Reduced expression and defective modulation of TNF receptor/ligand family molecules on proB-ALL blasts. Klin Pädiatr 2008; 220: 353-357

24 Winterhalter S, Niehues T. TNF $\alpha$ - blocking agents or convential immunosuppressive drugs in the therapy of children with uveitis? - An evidence based approach. Klin Pädiatr 2008; 220: 342-347 